\title{
Efeitos adversos associados à aplicação de toxina botulínica na face: revisão sistemática com meta-análise
}

\author{
Adverse effects associated with facial application of botulinum toxin: \\ a systematic review with meta-analysis
}

\author{
Roberta Melissa Benetti Zaguí ${ }^{1}$ \\ Suzana Matayoshi² \\ Frederico Castelo Moura ${ }^{3}$
}

Trabalho realizado no Departamento de Oftalmologia da Faculdade de Medicina da Universidade de São Paulo - USP - São Paulo (SP) - Brasil.

${ }^{1}$ Estagiária do Setor de Plástica Ocular do Departamento de Oftalmologia da Faculdade de Medicina da Universidade de São Paulo - USP - São Paulo (SP) - Brasil.

${ }^{2}$ Chefe do Setor de Plástica Ocular do Departamento de Oftalmologia da Faculdade de Medicina da USP - São Paulo (SP) - Brasil.

${ }^{3}$ Doutor, Médico do Departamento de Oftalmologia da Faculdade de Medicina da USP - São Paulo (SP) - Brasil.

Endereço para correspondência: Frederico Castelo Moura. Rua Artur de Azevedo, 166/151 E - São Paulo (SP) CEP 05404-000

E-mail: fredcastelo@terra.com.br

Recebido para publicação em 03.02.2008

Última versão recebida em 25.08.2008

Aprovação em 14.10.2008

Os autores não têm interesse ou apoio financeiro relacionado ao objeto de estudo deste manuscrito.

\section{RESUMO}

Objetivo: Verificar a ocorrência dos efeitos adversos relacionados ao uso da toxina botulínica na face, por meio de revisão sistemática, usando metaanálise. Métodos: Foi realizada pesquisa eletrônica de artigos publicados no MEDLINE e Cochrane Library até setembro de 2007. A ocorrência dos efeitos adversos foi verificada pela frequiência relativa para os relatos de casos e do risco relativo para os estudos randomizados. A heterogeneidade foi avaliada pelo teste $\mathrm{Q}$ e pelo índice $\mathrm{I}^{2}$. Foi utilizado o teste de significância de Egger para identificar viés de publicação e a análise de sensibilidade para verificar o efeito dos viéses de publicação. Resultados: Oito estudos randomizados e treze relatos de casos preencheram os critérios de inclusão. Na revisão sistemática dos relatos de casos, 1.003 pacientes foram estudados, dos quais $182(18,14 \%)$ apresentaram efeitos adversos. $\mathrm{O}$ efeito adverso mais freqüente foi a ptose palpebral ocorrendo em $34(3,39 \%)$ pacientes dos relatos de casos. Na meta-análise dos estudos randomizados, o risco relativo global para os efeitos adversos como cefaléia, ptose palpebral, reação local e infecção foi 1,07, 3,25, 0,99 e 0,94, respectivamente. Para todas as comparações, o valor de $\mathrm{P}$ foi maior que 0,05. Conclusão: O efeito adverso mais freqüente e que apresentou o maior risco relativo relacionado ao uso da toxina botulínica na face foi a ptose palpebral. A padronização dos efeitos adversos relatados é necessária a fim de melhor estabelecer as características dos mesmos.

Descritores: Toxina botulínica/efeitos adversos; Blefaroptose/etiologia; Revisão; Metanálise

\section{INTRODUÇÃO}

Toxina botulínica é uma exotoxina produzida pelo Clostridium botulinum, uma bactéria Gram-positiva e anaeróbica. Existem oito sorotipos de bactérias (A, B, C alpha, C beta, D, E, F e G) que produzem sete exotoxinas distintas; o tipo A, B e E são os mais comumente associados ao botulismo em humanos ${ }^{(1)}$. A via final da ação da toxina é impedir a liberação da acetilcolina na junção neuromuscular dos músculos estriados produzindo, assim, uma denervação química e, conseqüentemente, paralisia muscular ${ }^{(2)}$.

Nas últimas três décadas, a toxina botulínica tem sido empregada para fins terapêuticos em inúmeras afecções ${ }^{(3)}$. A toxina botulínica do tipo A foi aprovada primeiramente em 1989 para o uso em estrabismo, blefaroespasmo e espasmo hemifacial. Em seguida, os tipos A e B foram aprovados para 
o tratamento de distonias cervicais e, recentemente, o tipo A foi aprovado para fins estéticos ${ }^{(4)}$.

Embora apresente alta margem de segurança, a utilização da toxina botulínica pode levar a efeitos adversos (EA's), associados ou não à denervação química. Estes efeitos se apresentam de forma moderada e transitória e com baixa freqüência, segundo a maioria dos trabalhos publicados, embora poucos destes enfatizem e caracterizem a ocorrência dos EA's ${ }^{(5)}$.

O objetivo deste trabalho foi verificar a ocorrência dos efeitos adversos relacionados ao uso facial da toxina botulínica através de revisão sistemática com meta-análise.

\section{MÉTODOS}

Esse trabalho obedeceu às diretrizes metodológicas para realização de uma referência sistemática com meta-análise ${ }^{(6-7)}$, estando de acordo com protocolo pré-determinado, baseado no manual Cochrane para revisões sistemáticas ${ }^{(8)}$.

Estratégia de busca para identificação dos estudos: foi realizada pesquisa nos bancos de dados virtuais Cochrane Library e MEDLINE, até setembro de 2007, utilizando as seguintes palavras-chave: botulinum toxin, botox, side effect e complication. Não houve restrição quanto ao idioma do estudo.

Critérios de inclusão e exclusão dos estudos: foram incluídos artigos com dados sobre a ocorrência de EA's após aplicação de toxina botulínica na face. Os artigos que preencheram os critérios de inclusão foram divididos em dois grupos: (1) estudo não-randomizado, envolvendo apenas pacientes e sem grupo controle (relatos de casos) e (2) estudo casocontrole, duplo-cego e randomizado (estudos randomizados). Foram excluídos os estudos utilizando a toxina botulínica em outro sítio que não a face; estudos onde o efeito adverso fosse o objetivo da aplicação facial (como em pacientes com paralisia facial ou com retração palpebral distiroidiana); estudos com utilização facial da toxina botulínica e sem descrição de efeitos colaterais; e as revisões de literatura sobre toxina botulínica. Relatos de casos (grupo 1) foram incluídos apenas quando apresentavam um número de participantes maior ou igual a cinco.

Avaliação da qualidade metodológica dos estudos randomizados: essa avaliação foi realizada apenas nos estudos inclusos no grupo 2. Para assegurar a qualidade metodológica, os autores avaliaram, de forma independente, os seguintes critérios com respectiva pontuação baseada em publicação prévia $^{(9)}$ : método de alocação, ocultação da alocação, suspensão do uso da toxina botulínica, cálculo do tamanho da amostra e validação externa (critérios de inclusão e exclusão). Foi expressa uma pontuação para cada trabalho de acordo com sua qualidade metodológica e uma pontuação mínima de quatro (em um total de oito pontos possíveis) foi exigida para a inclusão do trabalho no presente estudo. Divergências quanto à qualidade metodológica dos estudos randomizados foram resolvidas por consenso entre os autores.
Coleta dos dados: os dados foram extraídos de cada estudo incluso em ambos os grupos, pelos autores, de forma independente. No grupo 1 (relatos de casos), foram extraídos o total de pacientes tratados e o total de pacientes para cada EA relatado. No grupo 2 (estudos randomizados), foi extraído o total de pacientes tratados com a toxina e de controles tratados com o placebo e o total de indivíduos acometidos por cada EA relatado, tanto para o grupo dos pacientes, como para o dos controles. Devido à falta de padronização dos EA's, ocasionando inúmeros tipos de EA's com baixa frequiência de eventos, optou-se por formar dois diferentes grupos: (I) EA's associados à reação local (hiperemia, equimose, dor, parestesia, edema e acne); (II) EA's compatíveis com infecção (febre, faringite, sinusite e infecção das vias aéreas superiores). Além disso, enxaqueca e cefaléia foram consideradas o mesmo EA. A coleta de dados respeitou as orientações do Cochrane Eyes and Vision Group ${ }^{(10)}$ para desfecho dicotômico.

Análise estatística: para os dados dos estudos do grupo 1, foi analisada a frequiência relativa de cada EA relatado. Metaanálise foi realizada para os EA's (ou grupo de EA's) dos estudos randomizados do grupo 2. Risco relativo (RR) e intervalo de confiança de $95 \%$ (IC 95\%) foram calculados para cada estudo randomizado individualmente e, em seguida, para a análise combinada (RR global) dos estudos randomizados. Foram considerados significantes os valores de $\mathrm{P}<0,05$. Quando o RR apresentava valor igual a 1 , não havia associação da toxina botulínica com o EA; quando maior que 1, a toxina foi considerada fator de risco; e quando menor do que 1 foi considerada fator de proteção, ou seja, o EA ocorreu preferencialmente no grupo controle. Se algum estudo apresentou "zero" evento do EA para o grupo de pacientes ou de controles, 0,5 foi adicionado automaticamente pelo programa MIX $^{\circledR}$ (Meta-analysis with Interactive Explanation) tanto no numerador como no denominador de ambos os grupos do estudo. Se ambos os grupos de um mesmo estudo não apresentavam evento, o programa excluiu automaticamente o estudo em questão. A heterogeneidade estatística foi avaliada pelo teste $\mathrm{Q}$ de Cochran $\left(\mathrm{X}^{2}\right)$ com nível de $\mathrm{P}<0,1$ para aceitar a hipótese de heterogeneidade entre os estudos. Esse nível de P é uma forma de compensar o baixo poder do teste de heterogeneidade, principalmente quando o número de estudos envolvidos é pequeno $^{(11)}$. Além disso, o índice $\mathrm{I}^{2}$ com intervalo de confiança de $95 \%$ foi calculado para medir a extensão da heterogeneidade $^{(12)}$. Se o IC $95 \%$ conter o valor $0 \%$, a homogeneidade entre os estudos foi aceita mesmo que o valor de $\mathrm{I}^{2}$ não seja $0 \%{ }^{(13)}$. Quando a hipótese de heterogeneidade entre os estudos foi aceita, o modelo de efeito randômico foi adotado para estimar o RR global. Gráficos em funil e teste de significância de Egger ${ }^{(14)}$ foram utilizados para identificar viés de publicação. Análise de sensibilidade foi realizada com o intuito de verificar se o resultado combinado foi afetado ou não pelos estudos considerados outliers potenciais e, assim, avaliar o grau de confiança dos resultados da meta-análise. Para isso, foram analisados o gráfico Trim-and-Fill e um novo resulta- 
do do RR global (e IC 95\%) foi calculado após a análise de sensibilidade. O programa MIX $^{\circledast}$ v. 1.6 foi utilizado para os cálculos e a construção dos gráficos das meta-análises. Teste do qui-quadrado $\left(\mathrm{X}^{2}\right)$ foi utilizado para comparar variáveis epidemiológicas categóricas.

\section{RESULTADOS}

\section{Busca eletrônica e características dos estudos}

Das 2.054 publicações inicialmente identificadas na nossa busca eletrônica, 82 foram potencialmente relevantes e foram analisadas de forma mais detalhada, através dos critérios de inclusão e exclusão propostos. Após este processo de seleção, 21 estudos (13 relatos de casos ${ }^{(15-27)}$, não randomizados, não cegos e sem controle; e 8 ensaios clínicos randomiza$\operatorname{dos}^{(28-35)}$, satisfizeram os critérios de inclusão deste estudo. Detalhes do processo de inclusão e exclusão dos estudos estão dispostos na figura 1 . Todos os 21 estudos revisados utilizaram toxina botulínica tipo A.

Entre os 13 relatos de casos, 9 tinham como objetivo avaliar a eficácia da toxina botulínica no tratamento de rugas ou linhas faciais e 4 no tratamento de blefarospasmo essencial e/ou de espasmo hemifacial. Foram analisados 1.003 indivíduos destes relatos, sendo 795 (79,3\%) mulheres e 208 (20,7\%) homens $(\mathrm{P}<0,001)$. A média de idade foi 51,5 anos (variação entre 38 e 59 anos). O seguimento médio após aplicação da toxina botulínica foi 12,1 meses (0,5-56 meses). Alguns estudos não forneceram dados quanto à idade dos pacientes ou ao tempo de seguimento após aplicação.

Os seis estudos randomizados tinham como objetivo avaliar a eficácia da toxina botulínica no tratamento de rugas ou linhas faciais. Um total de 1.521 indivíduos analisados (1.116 pacientes e 405 controles), com 1.317 (86,6\%) mulheres e 204 $(13,4 \%)$ homens $(\mathrm{P}<0,001)$. A média de idade foi 44,8 anos para os pacientes e 46,2 para os controles $(\mathrm{P}=0,21)$. O seguimento médio após aplicação da toxina botulínica foi 4,25 meses (variação entre 3-6 meses). A qualidade metodológica dos estudos randomizados variou de $62 \%$ a $88 \%$ (Tabela 1 ).

\section{Efeitos adversos - relatos de casos}

A tabela 2 mostra a frequiência relativa dos EA's nos 13 relatos de casos. Dos 1.003 pacientes estudados, 178 $(17,74 \%)$ apresentaram EA's e ptose palpebral foi o efeito adverso mais comum, ocorrendo em 34 (3,39\%) pacientes.

\section{Efeitos adversos - estudos randomizados}

Após a coleta dos dados dos oito estudos randomizados inclusos no estudo, os efeitos adversos cefaléia e ptose palpe-

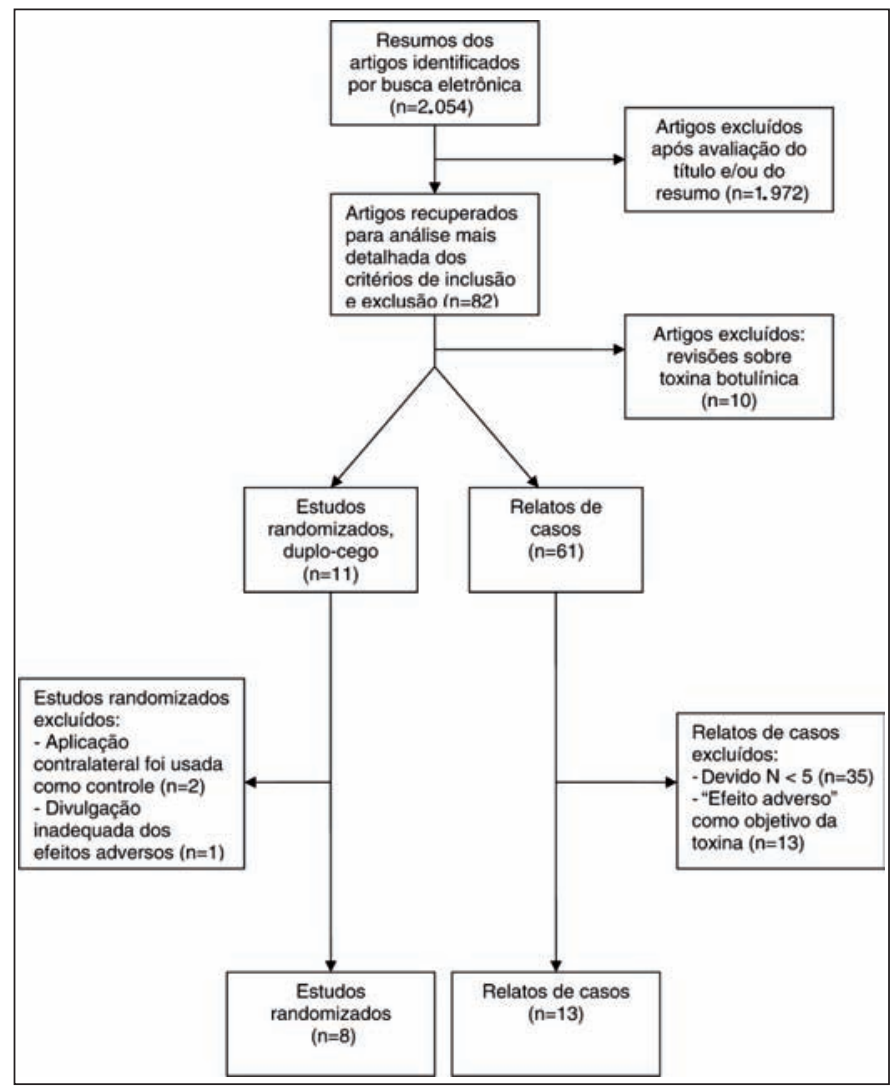

Figura 1 - Processo de inclusão e exclusão dos estudos para revisão sistemática com meta-análise

\begin{tabular}{|c|c|c|c|c|c|c|c|}
\hline \multirow[b]{2}{*}{ Estudo } & \multicolumn{5}{|c|}{ Itens da qualificação metodológica } & \multicolumn{2}{|c|}{ Pontuação } \\
\hline & A & B & C & D & $\mathbf{E}$ & Absoluta & Relativa \\
\hline Carruthers et al. $2007^{(34)}$ & 1 & 2 & 2 & 1 & 1 & 7 & $88 \%$ \\
\hline Monheit et al. 2007(35) & 1 & 2 & 1 & 1 & 1 & 6 & $75 \%$ \\
\hline Fagien et al. $2007^{(33)}$ & 2 & 2 & 2 & 0 & 1 & 7 & $88 \%$ \\
\hline Rzany et al. $2006^{(30)}$ & 1 & 2 & 1 & 0 & 1 & 5 & $62 \%$ \\
\hline Carruthers et al. $2003^{(28)}$ & 2 & 2 & 1 & 0 & 1 & 6 & $75 \%$ \\
\hline Carruthers et al. $2002^{(31)}$ & 2 & 2 & 1 & 1 & 1 & 7 & $88 \%$ \\
\hline \multicolumn{8}{|c|}{$\begin{array}{l}\text { Itens da qualificação metodológica [pontuação]: (A) alocação [2- randomização com metodologia definida; } 1 \text { - randomização sem metodologia definida; } 0 \text { - parcialmente } \\
\text { randomizado]; (B) ocultação da alocação [2- duplo-cego; } 1 \text { - simples-cego; 0- não-cego ou não definido]; (C) suspensão do uso da toxina botulínica [2- descrição do } \\
\text { número e razão das suspensões; } 1 \text { - descrição do número ou razão das suspensões; 0- número e razão não relatados]; (D) cálculo prévio do tamanho da amostra } \\
\text { [1- descrito previamente; } 0 \text { - não descrito] e (E) validação externa [1- critérios de inclusão e exclusão definidos; 0- critérios de inclusão e/ou exclusão não definidos] }\end{array}$} \\
\hline
\end{tabular}




\begin{tabular}{|lcc|}
\hline \multicolumn{3}{|l|}{ Tabela 2. Freqüência relativa dos efeitos adversos relacionados ao } \\
uso da toxina botulínica em $\mathbf{1 . 0 0 3}$ pacientes nos $\mathbf{1 3}$ relatos de casos \\
Efeitos adversos & N & $\%$ \\
Ptose palpebral & 34 & 3,39 \\
Olho seco & 23 & 2,29 \\
Edema local & 20 & 1,99 \\
Boca seca & 20 & 1,99 \\
Cefaléia & 16 & 1,59 \\
Paresia local & 11 & 1,09 \\
Equimose local & 8 & 0,79 \\
Eritema local & 7 & 0,69 \\
Ptose de supercílio & 6 & 0,59 \\
Diplopia & 6 & 0,59 \\
Sensação de peso local & 5 & 0,49 \\
Sangramento local & 5 & 0,49 \\
Melhora da cefaléia & 3 & 0,29 \\
Desvio de rima bucal & 3 & 0,29 \\
Alteração facial & 3 & 0,29 \\
Prurido local & 3 & 0,29 \\
Náusea & 2 & 0,19 \\
Estado gripal & 2 & 0,19 \\
Perda visual & 1 & 0,09 \\
\hline
\end{tabular}

bral, juntamente com os dois grupos de efeitos adversos (reação local e infecção), foram considerados para meta-análise. O modelo de efeito fixo foi utilizado após a constatação da homogeneidade entre os estudos.

Para o EA cefaléia, seis estudos foram considerados para a meta-análise (Figura 2-A). De um total de 1.411 indivíduos (1.061 pacientes e 350 controles), 152 (117 pacientes e 35 controles) apresentaram cefaléia após o tratamento, com um RR global de 1,07 ( $\mathrm{P}=0,68)$. Foi observado viés de publicação na análise do gráfico em funil, embora estatisticamente não significante (Egger $\mathrm{P}=0,44)$. No gráfico Trim-and-Fill, foram identificados dois estudos out-liers ${ }^{(29-30)}$ e o novo valor calculado para o RR global foi 1,04 (0,53-1,73).

Para o EA ptose palpebral, quatro estudos foram considerados para a meta-análise (Figura 2-B). De um total de 1.130 indivíduos (829 pacientes e 301 controles), 17 (todos pacientes) apresentaram ptose palpebral após o tratamento com toxina botulínica, com um RR global de 3,25 (P=0,11). Nenhum indivíduo do grupo controle apresentou ptose palpebral nesta análise. Não foi observado viés de publicação (Egger $\mathrm{P}=0,12$ ). Não foi identificado estudo outlier no gráfico Trim-and-Fill e assim, não houve alteração do valor do RR após a análise da sensibilidade.

Para o EA reação local, seis estudos foram considerados para a meta-análise (Figura 3-A). De um total de 884 indivíduos (634 pacientes e 250 controles), 45 (34 pacientes e 11 controles) apresentaram reação local após o tratamento, com um RR global de $0,99(\mathrm{P}=0,97)$. Foi observado viés de publicação na análise do gráfico em funil, embora estatisticamente não significante (Egger $\mathrm{P}=0,24)$. No gráfico Trim-and-Fill, foi identificado um estudo outlier ${ }^{(30)}$ e o novo valor calculado para o RR global foi $0,96(0,51-1,72)$.
Para o EA infecção, cinco estudos foram considerados para a meta-análise (Figura 3-B). De um total de 1.292 indivíduos (959 pacientes e 333 controles), 84 (64 pacientes e 20 controles) apresentaram infecção após o tratamento, com um RR global de $0,94(P=0,68)$. Não foi observado viés de publicação na análise do gráfico em Funil (Egger $\mathrm{P}=0,87$ ). Não foi identificado estudo outlier no gráfico Trim-and-Fill e por isso não houve alteração do valor do RR após a análise da sensibilidade.

\section{DISCUSSÃO}

Os autores têm conhecimento da rara utilização da revisão sistemática com meta-análise para avaliar a ocorrência de efeitos adversos relacionados a uma medicação e de sua freqüente utilização para verificar a eficácia terapêutica da mesma ${ }^{(6,36)}$. Sendo assim, este estudo foi desenvolvido devido à ausência de publicações dessa natureza e a possibilidade de identificação de um novo padrão de ocorrência dos EA's associados ao uso da toxina botulínica na face. Os resultados deste estudo mostraram que efeito adverso ocorre em, aproximadamente, $1 / 5(17,74 \%)$ dos indivíduos submetidos à toxina botulínica e que ptose palpebral é o EA mais associado ao uso da toxina botulínica, tanto nos relatos de casos $(3,39 \%)$ como nos estudos randomizados $(R R=3,25)$, embora estatisticamente não significante. Os outros três EA's avaliados na meta-análise dos estudos randomizados apresentaram valores de $R R$ próximos de um $(R R=1,0$; todas as comparações $\mathrm{P}<0,05)$ e, portanto, uma chance similar de ocorrer tanto após uso da toxina botulínica como do placebo.

Os valores estatisticamente não significantes do RR global nas meta-análises não podem ser considerados como uma evidência de ausência dos efeitos adversos uma vez que, se o tamanho da amostra for aumentado, uma significância poderá ser evidenciada. A amostra pode ser aumentada a partir de um número maior de estudos envolvidos na meta-análise, como a inclusão de estudos caso-controle não publicados em periódicos, embora estes dados não sejam tão rigorosamente avalia$\operatorname{dos}^{(37)}$. Provavelmente, a inclusão, ou não, de estudos não publicados seja a maior questão metodológica nas meta-análises e a principal fonte de viés de publicação. Além disso, a inclusão de indivíduos com idade inferior a 60 anos, não usuários de outras medicações e sem co-morbidades pode ter contribuído para uma subestimação dos efeitos adversos associados à aplicação facial da toxina botulínica.

Possivelmente, a ocorrência de ptose palpebral foi supervalorizada neste estudo devido à sua apresentação clínica evidente, enquanto outros efeitos adversos comumente dependem da informação do paciente. Assim, sintomas inespecíficos, como cefaléia ou reação infecciosa, podem ter sido omitidos por serem considerados irrelevantes e a ptose palpebral, cujo registro de ocorrência é dependente do examinador, se apresentaria com maior freqüência. Este fato pode ter sido o responsável pelo alto valor do RR da ptose palpebral em estudo prévio que encontrou RR de 6,99(31). 


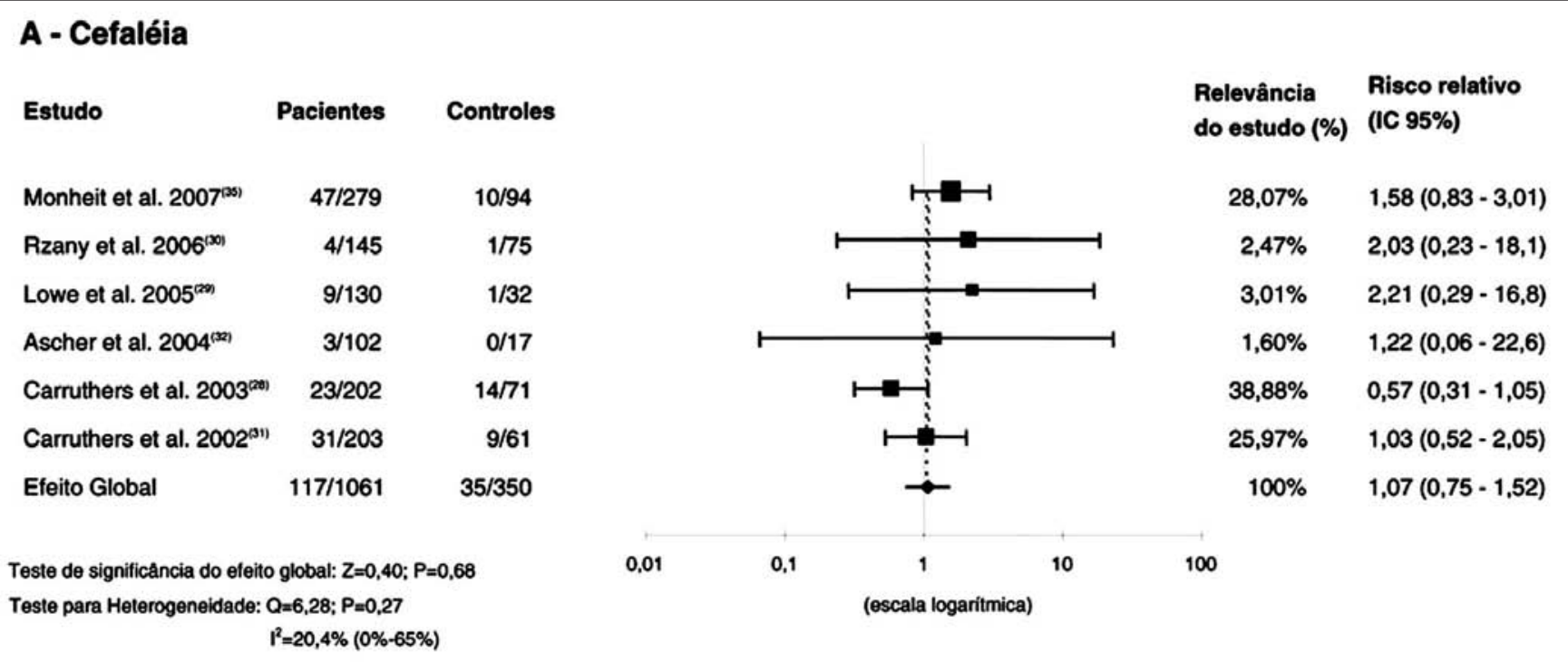

\section{B - Ptose palpebral}

$\begin{array}{lrr}\text { Estudo } & \text { Pacientes } & \text { Controles } \\ \text { Monheit et al. 2007 } & 3 / 279 & 0 / 94 \\ \text { Rzany et al. 2006 } & 1 / 145 & 0 / 75 \\ \text { Carruthers et al. 2003 } & 2 / 202 & 0 / 71 \\ \text { Carruthers et al. 2002 } & 11 / 203 & 0 / 61 \\ \text { Efeito global } & 17 / 829 & 0 / 301\end{array}$
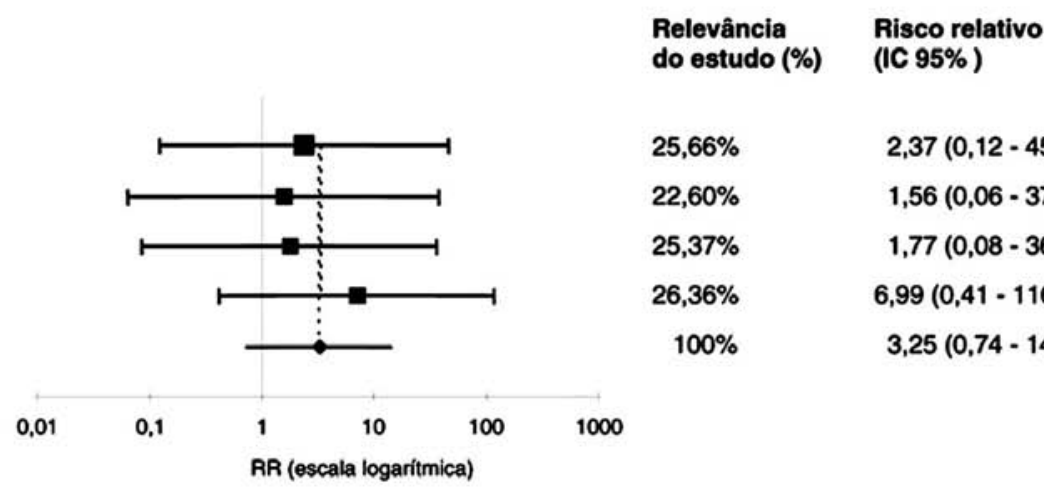

$25,66 \%$

$22,60 \%$

$25,37 \%$

$26,36 \%$

$100 \%$

$2,37(0,12 \cdot 45,5)$

$1,56(0,06-37,8)$

$1,77(0,08-36,5)$

$6,99(0,41 \cdot 116,9)$

$3,25(0,74-14,2)$

Teste de significância do efeito combinado: $Z=1,56 ; P=0,11$

Teste de Heterogeneidade: $Q=0,68 ; P=0,87$

$I^{2}=0 \%(0 \%-84 \%)$

Figura 2 - Risco relativo e intervalo de confiança de $95 \%$ (IC 95\%) dos efeitos adversos cefaléia (A) e ptose palpebral (B) associados à toxina botulínica nos estudos randomizados. Significância do efeito global e testes de heterogeneidade são mostrados.

A ausência de padronização metodológica para a definição dos efeitos adversos, tanto nos estudos randomizados como nos relatos de casos, foi uma importante limitação do presente estudo. Por isso, alguns efeitos adversos foram considerados como o mesmo (como cefaléia e enxaqueca) e outros foram aglomerados em grupos no intuito de uniformizar os dados e aumentar o poder da análise estatística através do aumento do número de eventos ${ }^{(38)}$, embora esta medida possa induzir a um viés na interpretação dos resultados. Alguns efeitos adversos da toxina botulínica observados na prática oftalmológica, como lagoftalmo, olho seco e ceratite puntata superficial ${ }^{(39)}$, não foram citados nos trabalhos analisados, pos- sivelmente devido à ausência de uma avaliação oftalmológica adequada da superfície ocular. Esse relato inadequado dos EA's também foi descrita como limitação em prévio estudo ${ }^{(5)}$.

Outra importante limitação metodológica foi a heterogeneidade clínica dos estudos randomizados em relação à dose da toxina botulínica aplicada nos pacientes, ao número de aplicações, aos sítios anatômicos e ao tempo de seguimento. A indicação do uso da toxina botulínica (se terapêutica ou cosmética) é outra possível fonte de heterogeneidade clínica tendo em vista a utilização de doses maiores de toxina botulínica para fins terapêuticos (hiperidrose, distonia cervical, espasticidade associada a acidente vascular cerebral, entre ou- 


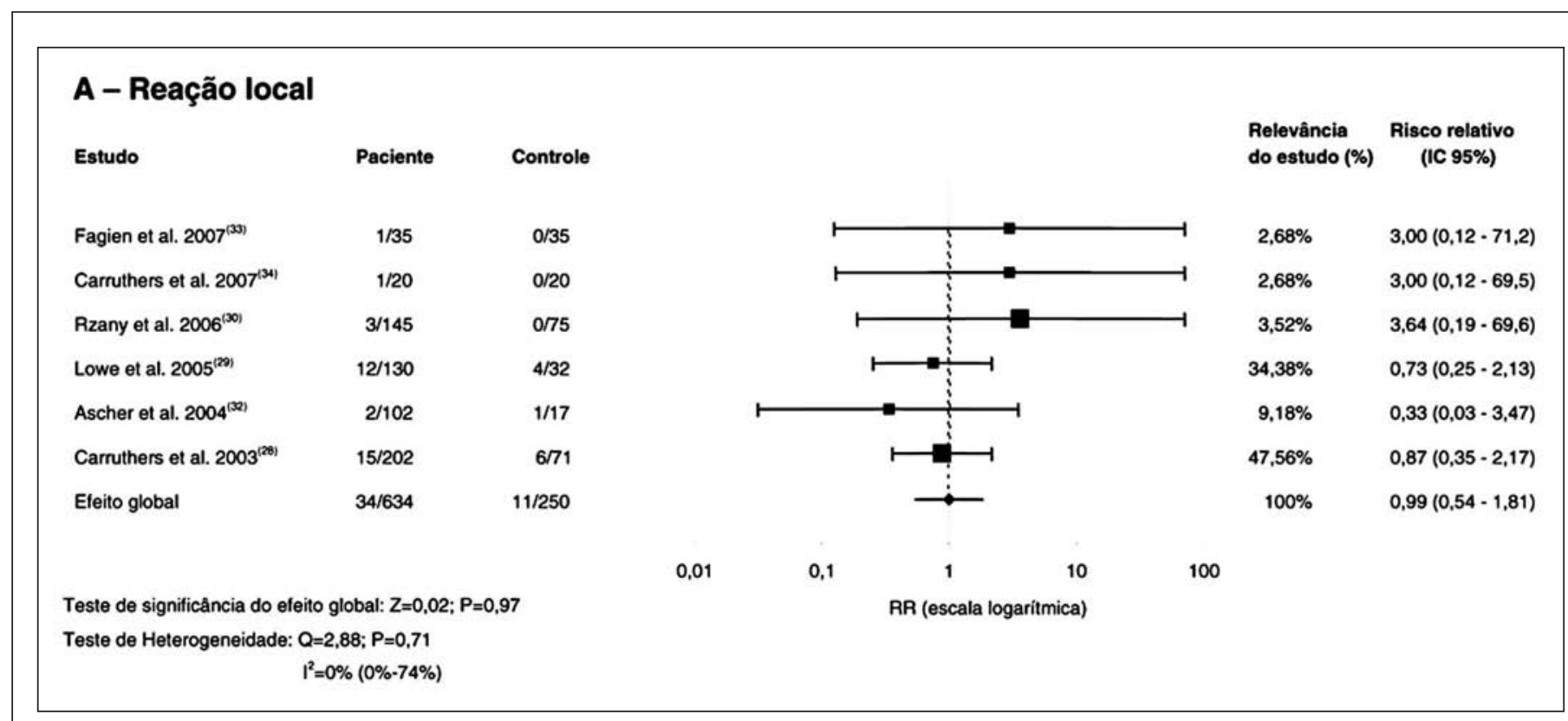

\section{B - Infecção}

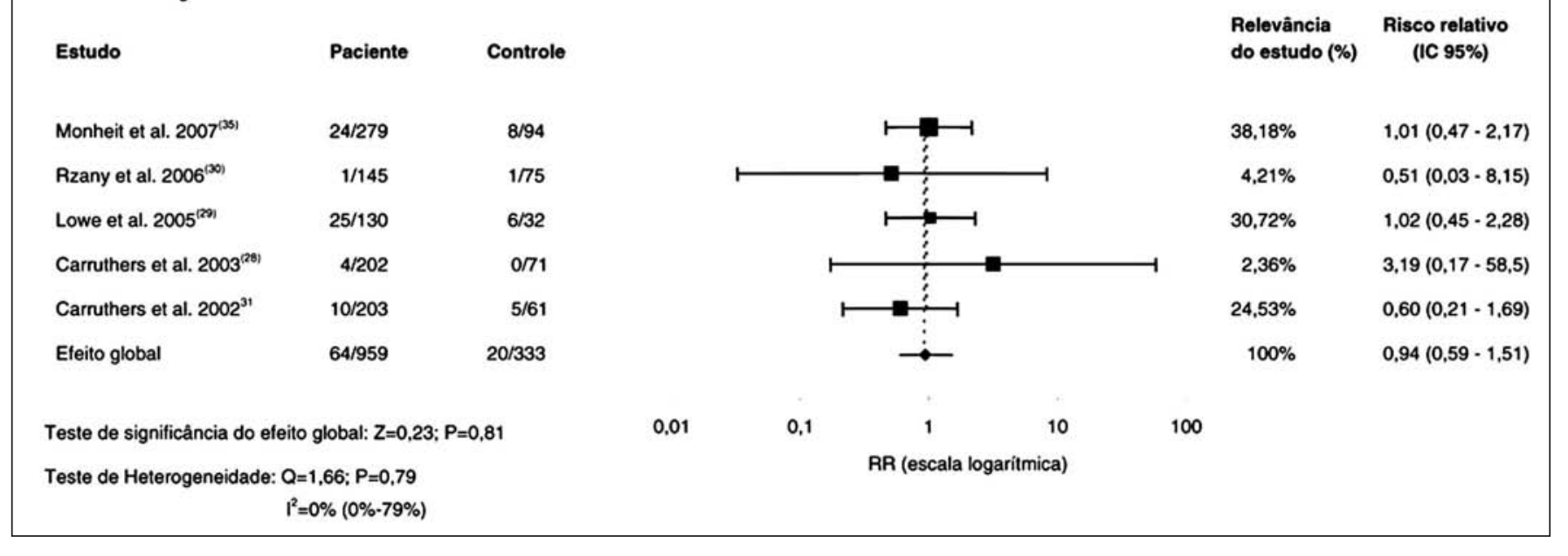

Figura 3 - Risco relativo e intervalo de confiança (IC 95\%) dos efeitos adversos reação local (A) e infecção (B) associados à toxina botulínica nos estudos randomizados. Significância do efeito global e testes de heterogeneidade são mostrados.

tros). Estudo realizado a partir de dados do $\mathrm{FDA}^{(5)}$ mostrou que os EA associados ao uso terapêutico da toxina botulínica são mais freqüentes e mais graves (como convulsão e morte) do que os associados ao uso cosmético e que podem estar relacionados às altas doses, à doença de base ou uso concomitante de outra medicação. No presente estudo, foram analisados artigos com indicação terapêutica (blefarospasmo essencial e espasmo hemifacial) e cosmética (rugas e linhas faciais) relacionada à face com intuito de reduzir este tipo de viés, pois apresentam doses recomendadas similares ${ }^{(4)}$. Assim, em vista da heterogeneidade clínica dos estudos analisados, inferências sobre o risco de EA após a aplicação da toxina botulínica devem ser cautelosas.
Todas as quatro meta-análises apresentaram homogeneidade estatística entre os estudos randomizados envolvidos ( $\mathrm{P}>0,1$; para todas as comparações), embora o resultado do índice $\mathrm{I}^{2}$ do EA cefaléia tenha sugerido leve heterogeneidade, possivelmente, relacionada às duas publicações outliers $^{(29-30)}$ envolvidas na meta-análise deste EA. Essas duas publicações apresentaram menor relevância para a meta-análise e os valores de RR individual mais distante do RR global. Publicação outlier ${ }^{(30)}$ também foi identificada na análise do EA reação local e este mesmo estudo apresentou a mais baixa qualidade entre os oito estudos randomizados e foi um dos dois outliers da análise do EA cefaléia. A correção do viés de publicação das duas metaanálises resultou em novos valores de RR global, embora próxi- 
mos dos valores do RR global inicial. Assim, os resultados das meta-análises (efeito global) do presente estudo são fidedignos mesmo na presença de viés de publicação.

Em conclusão, os resultados do presente estudo sugerem que a ocorrência de ptose palpebral está associada ao uso da toxina botulínica, embora estatisticamente não significante. O RR global dos outros efeitos adversos apresentou valores próximos de 1,0 sugerindo mínima ou ausência de associação com o uso de toxina botulínica e que a ocorrência destes EA's pode estar associada a outros parâmetros.

\section{ABSTRACT}

Purpose: To estimate the occurrence of adverse effects associated with the use of botulinum toxin by performing a systematic review and meta-analysis. Methods: A systematic literature search of MEDLINE and Cochrane Library was conducted until September 2007. The occurrence of the adverse effects was estimated of the relative frequency for case report and of the relative risk for randomized trials. Heterogeneity was evaluated with the $\mathrm{Q}$ test and $\mathrm{I}^{2}$ index. Egger's significance test was used to identify the publication bias. Sensitivity analysis was performed to evaluate the effect of the publication biases. Results: Eight randomized trials and thirteen case reports filled the inclusion criteria. In the systematic review of case reports, 1,003 subjects were studied and $182(18.14 \%)$ showed adverse effects. Eyelid ptosis was the most frequent adverse effect, presenting in 34 (3.39\%) patients of the case reports. In the meta-analysis of randomized trials, the overall relative risk for adverse effects as headache, eyelid ptosis, local reaction and infection was respectively: $1.07,3.25,0.99$ and 0.94 . For all comparisons, $\mathrm{P}$ values were greater than 0.05. Conclusion: Ptosis was the most frequent adverse effect and showed the higher relative risk associated with botulinum toxin. It is necessary to perform a pattern of adverse effects related for better understanding the relationship between adverse effects and use of botulinum toxin.

Keywords: Botulinum toxin/adverse effects; Blepharoptosis/ etiology; Review; Meta-analysis

\section{REFERÊNCIAS}

1. Klein AW. Contraindications and complications with the use of botulinum toxin. Clin Dermatol. 2004;22(1):66-75.

2. Klein AW. Complications and adverse reactions with the use of botulinum toxin. Dis Mon. 2002;48(5):336-56. Review.

3. Dutton JJ, Fowler AM. Botulinum toxin in ophthalmology. Surv Ophthalmol. 2007;52(1):13-31. Review.

4. Glogau RG. Review of the use of botulinum toxin for hyperhidrosis and cosmetic purposes. Clin J Pain. 2002;18(6 Suppl):S191-7.

5. Coté TR, Mohan AK, Polder JA, Walton MK, Braun MM. Botulinum toxin type A injections: adverse events reported to the US Food and Drug Administration in therapeutic and cosmetic cases. J Am Acad Dermatol. 2005;53(3): 407-15. Comment in: J Am Acad Dermatol. 2005;53(6):1080-2.

6. Egger M, Smith GD, Phillips AN. Meta-analysis: principles and procedures. BMJ. 1997;315(7121):1533-7.
7. Pogue J, Yusuf S. Overcoming the limitations of current meta-analysis of randomised controlled trials. Lancet. 1998;351(9095):47-52.

8. Higgins JPT, Green S, editors. Cochrane Handbook for Systematic Reviews of Interventions. Version 4.2.5 [updated May 2005]. Chichester, England: John Wiley \& Sons Ltd; Appendix 8a:215-229. [cietd 2006 Apr 24]. Available at: http://www.cochrane.org/ resources/handbook/hbook.htm.

9. Cheng JW, Wei RL, Cai JP, Xi GL, Zhu H, Li Y, Ma XY. Efficacy of different intraocular lens materials and optic edge designs in preventing posterior capsular opacification: a meta-analysis. Am J Ophthalmol. 2007;143(3):428-36.

10. Cochrane eyes and Vision Group. Cochrane Eyes and Vision Group (CEVG) systematic review activity on posterior segment treatments. Community Eye Health. 2006;19(57):11.

11. Bland M. Meta-analysis: methods for quantitative data synthesis. University of York, Heslington, York, UK. 2006. [Internet]. Available from: http:// www.users.york.ac.uk/ mb55/msc/systrev/meta_text.pdf

12. Huedo-Medina TB, Sánchez-Meca J, Marín-Martínez F, Botella J. Assessing heterogeneity in meta-analysis: Q statistic or I2 index? Psychol Methods. 2006;11(2):193-206.

13. Higgins JP, Thompson SG. Quantifying heterogeneity in a meta-analysis Stat Med. 2002;21(11):1539-58.

14. Egger M, Davey Smith G, Schneider M, Minder C. Bias in meta-analysis detected by a simple, graphical test. BMJ. 1997;315(7109):629-34.

15. Ahn KY, Park MY, Park DH, Han DG. Botulinum toxin A for the treatment of facial hyperkinetic wrinkle lines in Koreans. Plast Reconstr Surg. 2000; 105(2):778-84.

16. Carruthers A, Bogle M, Carruthers JD, Dover JS, Arndt KA, Hsu TS, Poirier J. A randomized, evaluator-blinded, two-center study of the safety and effect of volume on the diffusion and efficacy of botulinum toxin type $\mathrm{A}$ in the treatment of lateral orbital rhytides. Dermatol Surg. 2007;33(5):567-71.

17. Carruthers A, Carruthers J, Cohen J. A prospective, double-blind, randomized, parallel- group, dose-ranging study of botulinum toxin type a in female subjects with horizontal forehead rhytides. Dermatol Surg. 2003;29(5):461-7.

18. Defazio G, Abbruzzese G, Girlanda P, Vacca L, Currà A, De Salvia R, et al. Botulinum toxin A treatment for primary hemifacial spasm: a 10-year multicenter study. Arch Neurol. 2002;59(3):418-20.

19. Flynn TC, Carruthers JA, Carruthers JA. Botulinum-A toxin treatment of the lower eyelid improves infraorbital rhytides and widens the eye. Dermatol Surg. 2001;27(8):703-8.

20. Heckmann M, Schön-Hupka G. Quantification of the efficacy of botulinum toxin type A by digital image analysis. J Am Acad Dermatol. 2001;45(4):508-14.

21. Hexsel DM, De Almeida AT, Rutowitsch M, De Castro IA, Silveira VL, Gobatto DO, et al. Multicenter, double-blind study of the efficacy of injections with botulinum toxin type A reconstituted up to six consecutive weeks before application. Dermatol Surg. 2003;29(5):523-9; discussion 529.

22. Niamtu $\mathrm{J} 3^{\text {rd }}$. Botulinum toxin A: a review of 1,085 oral and maxillofacial patient treatments. J Oral Maxillofac Surg. 2003;61(3):317-24.

23. Oyama H, Ikeda A, Inoue S, Nakashima Y, Shibuya M. Local injection of botulinum toxin type A for hemifacial spasm. Neurol Med Chir (Tokyo) 2002;42(6):245-8; discussion 248-9.

24. Park YC, Lim JK, Lee DK, Yi SD. Botulinum a toxin treatment of hemifacial spasm and blepharospasm. J Korean Med Sci. 1993;8(5):334-40.

25. Pribitkin EA, Greco TM, Goode RL, Keane WM. Patient selection in the treatment of glabellar wrinkles with botulinum toxin type A injection. Arch Otolaryngol Head Neck Surg. 1997;123(3):321-6.

26. Schellini SA, Matai O, Igami TZ, Padovani CR, Padovani CP. Blefarospasmo essencial e espasmo hemifacial: características dos pacientes, tratamento com toxina botulínica A e revisão da literatura. Arq Bras Oftalmol. 2006; 69(1):23-6.

27. West TB, Alster TS. Effect of botulinum toxin type A on movement-associated rhytides following CO2 laser resurfacing. Dermatol Surg. 1999;25(4):259-61.

28. Carruthers JD, Lowe NJ, Menter MA, Gibson J, Eadie N; Botox Glabellar Lines II Study Group. Double-blind, placebo-controlled study of the safety and efficacy of botulinum toxin type A for patients with glabellar lines. Plast Reconstr Surg. 2003;112(4):1089-98.

29. Lowe NJ, Ascher B, Heckmann M, Kumar C, Fraczek S, Eadie N; Botox Facial Aesthetics Study Team. Double-blind, randomized, placebo-controlled, dose-response study of the safety and efficacy of botulinum toxin type A in subjects with crow's feet. Dermatol Surg. 2005;31(3):257-62.

30. Rzany B, Ascher B, Fratila A, Monheit GD, Talarico S, Sterry W. Efficacy and safety of 3- and 5-injection patterns (30 and $50 \mathrm{U}$ ) of botulinum toxin A (Dysport) for the treatment of wrinkles in the glabella and the central forehead region. Arch Dermatol. 2006;142(3):320-6. Comment in: Arch Dermatol. 2006;142(3):362-4. 
31. Carruthers JA, Lowe NJ, Menter MA, Gibson J, Nordquist M, Mordaunt J, Walker P, Eadie N; BOTOX Glabellar Lines I Study Group. A multicenter, double-blind, randomized, placebo-controlled study of the efficacy and safety of botulinum toxin type A in the treatment of glabellar lines. J Am Acad Dermatol. 2002;46(6):840-9.

32. Ascher B, Zakine B, Kestemont P, Baspeyras M, Bougara A, Santini J. A multicenter, randomized, double-blind, placebo-controlled study of efficacy and safety of 3 doses of botulinum toxin $\mathrm{A}$ in the treatment of glabellar lines. J Am Acad Dermatol. 2004;51(2):223-33. Erratum in: J Am Acad Dermatol. 2005;52(1):156. Comment in: J Am Acad Dermatol. 2005;53(2):364-5.

33. Fagien S, Cox SE, Finn JC, Werschler WP, Kowalski JW. Patient-reported outcomes with botulinum toxin type A treatment of glabellar rhytids: a double-blind, randomized, placebo-controlled study. Dermatol Surg. 2007; 33(1 Spec No.):S2-9.

34. Carruthers J, Carruthers A. Botulinum toxin type A treatment of multiple upper facial sites: patient-reported outcomes. Dermatol Surg. 2007;33(1 Spec No.): S10-7.

35. Monheit G, Carruthers A, Brandt F, Rand R. A randomized, double-blind, placebo- controlled study of botulinum toxin type A for the treatment of glabellar lines: determination of optimal dose. Dermatol Surg. 2007;33(1 Spec No.):S51-9.

36. Mittmann N, Liu BA, Knowles SR, Shear NH. Meta-analysis and adverse drug reactions. CMAJ. 1999;160(7):987-8.

37. Tissot MCRG, Louzã Neto MR, Elkis H. Os antipsicóticos de nova geração e suas meta-análises. Rev Psiquiatr Clín (Sao Paulo). 2003;30(6):229-32.

38. Bongartz T, Sutton AJ, Sweeting MJ, Buchan I, Matteson EL, Montori V. Anti-TNF antibody therapy in rheumatoid arthritis and the risk of serious infections and malignancies: systematic review and meta-analysis of rare harmful effects in randomized controlled trials. JAMA. 2006;295(19):2275-85. Erratum in: JAMA. 2006;295(21):2482. Comment in: ACP J Club. 2006;145(3):65. Arch Dermatol. 2007;143(3):405-6. JAMA. 2006;296(18):2201-2; author reply 2203-4. JAMA. 2006;296(18):2201; author reply 2203-4. JAMA. 2006; 296(18):2202; author reply 2203-4. JAMA. 2006;296(18):2203; author reply 2203-4. JAMA. 2006;296(18):2205.

39. Northington ME, Huang CC. Dry eyes and superficial punctate keratitis: a complication of treatment of glabelar dynamic rhytides with botulinum exotoxin A. Dermatol Surg. 2004;30(12 Pt2):1515-7. 